\title{
Securing the Border from Invasives: Robust Inspections under Severe Uncertainty
}

\author{
L. Joe Moffitt, ${ }^{1}$ John K. Stranlund, ${ }^{1}$ and Craig D. Osteen ${ }^{2}$ \\ ${ }^{1}$ Department of Resource Economics, University of Massachusetts at Amherst, 80 Campus Center Way, Amherst, MA 01003, USA \\ ${ }^{2}$ United States Department of Agriculture, Economic Research Service in Washington, DC 20036, USA
}

Correspondence should be addressed to L. Joe Moffitt, moffitt@resecon.umass.edu

Received 16 March 2010; Accepted 25 May 2010

Academic Editor: Yew-Kwang Ng

Copyright ( $) 2010$ L. Joe Moffitt et al. This is an open access article distributed under the Creative Commons Attribution License, which permits unrestricted use, distribution, and reproduction in any medium, provided the original work is properly cited.

Two important features of agricultural quarantine inspections of shipping containers for invasive species at U.S. ports of entry are the general absence of economic considerations and the severe uncertainty that surrounds invasive species introductions. In this article, we propose and illustrate a method for determining an inspection monitoring protocol that addresses both issues. An inspection monitoring protocol is developed that is robust in maximizing the set of uncertain outcomes over which an economic performance criterion is achieved. The framework is applied to derive an alternative to Agricultural Quarantine Inspection (AQI) for shipments of fruits and vegetables as currently practiced at ports of entry in the United States.

\section{Introduction}

At United States ports of entry, the contents of air, maritime, truck, and rail cargo, as well as air passenger baggage, vehicles, and mail are subject to Agricultural Quarantine Inspection (AQI) by the United States Department of Homeland Security (DHS), Customs and Border Protection officials. The purpose of AQI is to help ensure that United States agriculture is protected from accidentally or intentionally introduced pests and diseases, including the possibility of agroterrorism. In general, current practice for inspecting cargo shipments of fruits and vegetables at United States ports is based on inspecting $2 \%$ of the items in a container for the presence of pests, with some allowances for the size, contents, and origin of the container (United States Department of Agriculture [1]).

Although simple to apply, this inspection rule appears not to have any economic content. That is, it does not consider the costs of inspections or the losses of failing to prevent an invasive species from entering the country, nor does it account for the severe uncertainty associated with infestations in shipping containers and the potential losses from introductions of poorly understood or surreptitiously introduced invasive species. In this paper, we propose an alternative decision criterion for determining inspection probabilities that incorporates economic considerations with particular emphasis on the severe uncertainties of pest introductions and damage.

With probability distributions over invasive species introductions and their impacts, one could cast the problem of determining optimal inspection rules in the familiar terms of risk analysis. A well-known implementation of this basic approach to decision making under risk is the expected utility model, where outcomes under different states of nature are weighted by probabilities when ranking actions. If probability distributions are not objectively known, the subjective expected utility model, where it is assumed that decision makers can develop subjective probability estimates and rank choices, is a well-known alternative. In the latter case, subjective probabilities are assumed either known by the decision maker or estimable using appropriate assessment procedures.

As already noted, it would be relatively straightforward to specify inspection rules that balance the costs of inspections against the expected benefits of preventing introductions of well-understood pests with well-behaved introduction pathways for which sufficient data are available to estimate probability distributions and impacts or for which subjective assessments are feasible. However, for very poorly-understood or unknown organisms, a risk-based 
approach can require information that policy makers may not possess and may have difficulty obtaining within a timeframe that is useful. For example, the likelihood of intentional introductions of invasive species can cast a shadow over the potential of both objective and subjective probability measures. In fact, biosecurity concerns have become an important consideration in many surveillance contexts and tend to make use of both historical data and expert opinion problematic in risk assessment. In some areas of economic decision making, including the management of invasive species, it is often difficult to interpret and rely on probability distributions associated with what may be onetime, uncertain events. Consequently, concerns about the usefulness of risk assessment in the management of invasive species are evident among researchers and practitioners alike (Moffitt and Osteen [2]). Moreover, even when risk assessment is thought to provide at least some useful information to a decision maker, decision criteria that are not based on vaguely understood probability functions can provide complementary information, for example, by elucidating the robustness of particular decisions to errors in the choice of distribution functions (see e.g., Stranlund and Ben-Haim [3]). For these reasons, nonprobabilistic decision criteria have been the subject of increased interest in the economics of surveillance realm.

Several approaches have been developed to analyze decision making in uncertain environments. These approaches include application of the maximin, maximax, Laplace, and Hurwicz criteria (Render et al. [4]). Each of these criteria can be interpreted in the context of a traditional decision problem with actions $A_{i}$, uncertain events $E_{j}$, and payoffs $V\left(A_{i}, E_{j}\right)$. The maximin optimal decision is found as the solution to $\operatorname{Maximize}_{\left(A_{i}\right)} \operatorname{Min}_{\left(E_{j}\right)} V\left(A_{i}, E_{j}\right)$. The maximax optimal decision is found as the solution to $\operatorname{Maximize}_{\left(A_{i}\right)} \operatorname{Max}_{\left(E_{j}\right)} V\left(A_{i}, E_{j}\right)$. The Laplace optimal decision is found as the solution to $\operatorname{Maximize}_{\left(A_{i}\right)} \sum_{j} V\left(A_{i}, E_{j}\right)$. The Hurwicz optimal decision (given a value for $w \in[0,1]$ ) is found as the solution to $\operatorname{Maximize}_{\left(A_{i}\right)}\left(w \operatorname{Max}_{\left(E_{j}\right)} V\left(A_{i}, E_{j}\right)+(1-\right.$ w) $\left.\operatorname{Min}_{\left(E_{j}\right)} V\left(A_{i}, E_{j}\right)\right)$. The first two of these approaches represent polar extremes in terms of optimism and pessimism while the latter two require information similar to probabilities to be applied. Similarly, quantification of other notions related to uncertainty such as ignorance and surprise have also required the specification of functions confined to the unit interval (Katzner [5]; Horan et al., [6]).The notion of ambiguity lies in the middle ground between risk and uncertainty. If the likelihood of uncontrolled events can be determined up to a convex set (e.g., ranges of probability values are known), then there is said to be ambiguity about the risks associated with a decision. For such cases, a decision criterion known as maxmin expected utility (Gilboa and Schmeidler [7]) suggests maximizing the minimum expected utility where the expectation is taken over the convex set.

Other decision theory research has focused on the notion of robustness in decision making under uncertainty, but without any information on probabilities. Ben-Haim [8] has developed a new approach known as information-gap (infogap) decision theory, which he designed for cases in which probability distributions for uncontrolled events are not available. The essence of info-gap analysis is the pursuit of decisions that are robust in the sense that, roughly speaking, they maximize the range of uncertainty in the decision environment within which the decision maker is certain to achieve a specified performance requirement. One decision is more robust than another if the range of uncertainty under which the performance requirement is met is larger. Given a performance criterion, a robust decision gives the decision maker maximum confidence that his or her performance criterion will be met.Info-gap decision theory is increasingly applied to real-world applications where probabilities or a convex set of probabilities are hard to identify but acceptable performance is not. Applications include, but are not limited to, financial risk assessment (Ben-Haim [9]), search behavior in animal foraging models (Carmel and Ben-Haim [10]), policy decisions in marine reserve design (Halpern et al. [11]), natural resource conservation decisions (Moilanen and Wintle [12]), inspection decisions by port authorities to detect terrorist weapons (Moffitt et al. [13]) and invasive species (Moffitt et al. [14]; Moffitt et al. [15]; Davidovitch et al. [16]; Rout et al. [17]; Burgman et al. [18]; Carrasco et al. [19]; Yemshanov et al. [20]), the choice of environmental policies (Stranlund and Ben-Haim [3]), and engineering model-testing (Vinot et al. [21]).

We adopt Ben-Haim's approach to the problem of determining robust inspection protocols for detecting invasive species in imported agricultural goods. In this problem, we are uncertain about the likelihood of the presence of an invasive species in the goods being inspected and the economic impact of inspection failure. Nevertheless, we seek an inspection protocol to maximize the set of uncertain outcomes over which the expected loss from an introduction plus the cost of inspections will not exceed a critical value. While this approach can lead to an optimal decision, that is, different from alternative decision criteria (e.g., expected utility maximization), the relationship to alternatives will depend on the critical value, referred to in the info-gap literature, as the performance requirement that is implemented, as well as parameters associated with the alternatives. Since the decision maker chooses a performance requirement, this approach does not result in a "worst-case" decision, unless the decision maker desires one (e.g., by using the maximin criterion), and is not necessarily more conservative than the expected utility approach. The decision maker can choose an optimistic performance requirement that results in low robustness. Alternatively, a risk averse expected utility maximizer may prefer a decision that would be regarded as "more conservative" than that chosen under Ben-Haim's approach if the performance requirement under the latter approach is not too demanding. This approach is chosen in recognition of the myriad uncertainties present in this decision making realm.

The economic literature on invasive species is relatively recent. A bibliography of the economics of agricultural pest control covering the literature through 1980 does not contain a single reference to invasive species (Osteen et al. [22]). Some more recent studies have focused on the economics of managing invasive species once they have been introduced 
(e.g., White et al. [23]). From a similar perspective,Pimental et al. [24] provide both background and an economic perspective on invasive species introductions that have occurred in the United States.

However, there is a growing literature that has focused on the prevention of invasive species introductions and preparedness for these events, rather than retrospective analyses of introductions (e.g., Perrings et al. [25],Shogren [26], Brown et al. [27], Barbier and Shogren [28], Eiswerth and van Kooten [29], Endress [30], Horan et al. [6], Kaiser and Roumasset [31], Olson and Roy [32], Perrings et al. [33], and Settle and Shogren [34]). A portion of this literature has focused on border inspections to prevent introductions.(Shogren [26] provides a theoretical model of a policy maker who is charged with allocating resources to reducing the probability of an invasive species event (mitigation) and to reducing the adverse consequences of an introduction (adaptation). One could think of border inspections as part of the set of mitigation strategies, but Shogren is not explicit about this.) McAusland and Costello [35] present theoretical models of international trade to consider the simultaneous choices of a tariff and inspections to prevent the entry of infested commodities. Surkov et al. [36] focus on allocating fixed inspection resources across commodities and countries of origin to minimize the expected costs of introduced pests. They apply their model to inspections of chrysanthemum cuttings (Dendranthema grandiflora) imported to the Netherlands. Importantly, each of these works relies on known probability density functions, and hence, are models of risk rather than of uncertainty.

Moffitt et al. [14, 15] examine the inspection problem with info-gap models of uncertainty. Moffitt et al. [14] develop a robust sample size for a risk averse decision maker faced with inspecting a generic shipping problem in which a shipment may contain at most a single contaminated item. Moffitt et al. [15] evaluate the relative robustness of alternative inspection rules for a risk neutral decision maker when the number of contaminated items can vary, but they assume that the loss when an invasive pest gets past port inspections is known. We extend this work in two important directions. First, we allow several elements of the inspection problem to be uncertain including the number of contaminated items in a shipment, the costs of inspections, and potential losses due to inspection failure. Second, we use recently available unpublished data provided by the U.S. Department of Homeland Security to illustrate the potential of our model to determine robust inspection rules.

We demonstrate the utility of our approach by comparing robust inspection rules to the AQI $2 \%$ rule. We find that optimal inspection rules provide significant increases in robustness over the AQI rule over a wide range of feasible performance criteria. Moreover, robust inspection rules suggest significantly more scrutiny of incoming shipments than the AQI rule. This suggests a reallocation of federal resources to more intense inspections and away from efforts to deal with invasives that get through the inspection process.

The rest of the paper proceeds as follows. We present the model of choosing inspection rules that are maximally robust to the uncertainty in the problem of detecting invasive species at ports in the next section. In the third section we apply the model with data about shipments that are subject to AQI, costs of inspections, and funds allocated to deal with pest introduction and outbreaks. The third section also contains our comparison of robust inspection rules to the AQI $2 \%$ rule. We conclude in the fourth section.

\section{A Model of Robust Inspections for Invasive Species}

Suppose that a single containerized shipment selected for inspection at a maritime port of entry contains $N$ boxes. Inspection of $n$ of the boxes for the presence of invasives is intended to determine whether the contents of the shipment are infested. Inspection failure is defined to mean that a shipment is identified as not infested when it contains at least one infested box. Hence, under this definition, failure cannot occur if either there are no infested boxes in the shipment or if more than $N-n$ boxes in the shipment are infested. To simplify the analysis of the inspection protocol, we assume that if a box is inspected the presence of an invasive will be detected. Inspection failure-an undetected entry of an invasive species-generates a loss $L$. The loss due to inspection failure depends on which invaders are involved, how hard the invaders are to manage following inspection failure, and what the consequences of entry are. Because each of these elements can be highly uncertain, we regard $L$ as highly uncertain. Additionally, a linear function, $c n$, gives the cost of inspection where $c$ is a parameter reflecting a constant per box inspection cost which can depend on a number of factors and can also be regarded as uncertain. The inspection cost function need not be linear for the model development, but we assume that it is linear in our simulations in the next section. We also recognize that inspection strategies may involve significant fixed costs. These costs do not affect our analysis so we ignore them for simplicity.

Presuming the absence of evasive strategic invader behavior, if the number of infested boxes in a container is $s>0$, then the probability of inspection failure is given by the ratio of binomial coefficients

$$
\left(\begin{array}{c}
N-s \\
n
\end{array}\right) /\left(\begin{array}{l}
N \\
n
\end{array}\right),
$$

that is, the ratio of the number of possible samples of size $n$ which do not contain an infested box to the total number of possible samples of size $n$. If $s=0$, then the probability of inspection failure is also zero as just noted.

The number of infested boxes in a container, if any, is uncertain, as is the potential loss from an inspection failure, and perhaps marginal inspection costs. Define the set $\delta=$ $\{0,1,2, \ldots, N\}$ to depict the possible number of infested boxes in the container. Similarly let the set $\mathcal{L}=\{0,1,2, \ldots\}$ be potential losses (in dollars) associated with inspection failure, and let the set $\mathcal{C}=\{.01, .02, \ldots\}$ be the possible cost in dollars of inspecting a box. In defining the info-gap uncertainty model, we follow the approach of Ben-Haim and Hipel [37] in dealing with a discrete horizon of uncertainty. We first define the set $U=\& \times \mathcal{L} \times \mathcal{C}$ and define $\alpha$ to 
be any element of $U$ with cardinality denoted by $|\alpha|$. The info-gap uncertainty model $\mathcal{U}$ is defined as the following family of nested sets for any positive integer $h: \mathcal{U}(h)=\{\alpha \in$ $U:|\alpha| \leq h\}$, that is $\mathcal{Y}(h)$ is the set of all subsets of $U$ whose cardinality is no greater than $h$. cost is

For $s>0$ and a given $L$ and $c$, expected loss plus inspection

$$
\left[\left(\begin{array}{c}
N-s \\
n
\end{array}\right) /\left(\begin{array}{l}
N \\
n
\end{array}\right)\right] L+c n
$$

If $s=0$ given $c$, expected loss due to undetected entry plus inspection cost is $c n$. Hence, conditional on $N, n, s, L$, and $c$, expected loss due to undetected entry plus inspection cost can be expressed as

$E[$ Cost $\mid N, n, s, L, c]$

$$
= \begin{cases}c n, & \text { if } s=0, \\
{\left[\left(\begin{array}{c}
N-s \\
n
\end{array}\right) /\left(\begin{array}{l}
N \\
n
\end{array}\right)\right] L+c n,} & \text { if } 0<s \leq N .\end{cases}
$$

For $s>0$ and a given $L$ and $c$, the variance of loss plus inspection cost is

$$
\left[\left(\begin{array}{c}
N-s \\
n
\end{array}\right) /\left(\begin{array}{l}
N \\
n
\end{array}\right)\right]\left[1-\left(\begin{array}{c}
N-s \\
n
\end{array}\right) /\left(\begin{array}{l}
N \\
n
\end{array}\right)\right] L^{2}
$$

If $s=0$ the variance of loss due to undetected entry plus inspection cost is 0 . Hence, conditional on $N, n, s, L$, and $c$, the variance of loss due to undetected entry plus inspection cost can be expressed as

$$
\begin{aligned}
& \operatorname{Var}[\operatorname{Cost} \mid N, n, s, L, c] \\
& = \begin{cases}0, & \text { if } s=0, \\
{\left[\left(\begin{array}{c}
N-s \\
n
\end{array}\right) /\left(\begin{array}{c}
N \\
n
\end{array}\right)\right]} & \\
\times\left[1-\left(\begin{array}{c}
N-s \\
n
\end{array}\right) /\left(\begin{array}{c}
N \\
n
\end{array}\right)\right] L^{2}, & \text { if } 0<s \leq N .\end{cases}
\end{aligned}
$$

Our objective is to provide an inspection protocol that is as applicable as AQI in addition to being most robust in meeting a performance requirement. To maintain equivalent simplicity to AQI, both conditional expected cost and the conditional variance of cost are respecified in terms of a constant percentage of items subjected to physical inspection namely,

$$
\begin{aligned}
& E[\text { Cost } \mid p, N, s, L, c] \\
& = \begin{cases}c r(p N), & \text { if } s=0, \\
{\left[\left(\begin{array}{c}
N-s \\
r(p N)
\end{array}\right) /\left(\begin{array}{c}
N \\
r(p N)
\end{array}\right)\right] L+c r(p N),} & \text { if } 0<s \leq N,\end{cases} \\
& \operatorname{Var}[\operatorname{Cost} \mid p, N, s, L, c] \\
& = \begin{cases}0, & \text { if } s=0, \\
\left.\left[\begin{array}{c}
N-s \\
r(p N)
\end{array}\right) /\left(\begin{array}{c}
N \\
r(p N)
\end{array}\right)\right] & \\
\times\left[1-\left(\begin{array}{c}
N-s \\
r(p N)
\end{array}\right) /\left(\begin{array}{c}
N \\
r(p N)
\end{array}\right)\right] L^{2}, & \text { if } 0<s \leq N,\end{cases}
\end{aligned}
$$

where $p$ is percentage of items inspected and $r(\cdot)$ denotes rounding to the nearest integer.

We now use (6) to characterize expected costs and variance of the total annual shipments $T$ into a country. Let $f(N)$ be the annual relative frequency of containers of size $N$. Annual expected loss plus inspection cost for all shipments is

$$
M(p, s, L, c)=\sum_{N} E[\text { Cost } \mid p, N, s, L, c] \cdot f(N) \cdot T .
$$

Assuming independence of failure between shipments, the variance of annual loss plus inspection cost is

$$
V(p, s, L, c)=\sum_{N} \operatorname{Var}[\text { Cost } \mid p, N, s, L, c] \cdot f(N) \cdot T^{2} .
$$

Both $M(p, s, L, c)$ and $V(p, s, L, c)$ can be used to specify performance of a constant percentage inspection protocol like AQI. If performance is judged solely in terms of limiting expected loss from invasives plus inspection costs, then an inspection protocol is judged on its ability to satisfy $M(\cdot) \leq$ $M^{*}$, where $M^{*}$ is predetermined. If performance is also regarded as depending on limiting variability of expected loss plus inspection cost then an inspection protocol is judged with respect to $M(\cdot) \leq M^{*}$ and $V(\cdot) \leq V^{*}$, where $V^{*}$ is also predetermined.

Now let us define robustness with respect to possible performance criteria. If the performance criterion is a limit on $M^{*}$ on expected loss from invasives plus inspection cost then

$$
\hat{\alpha}(p)=\max \left\{h:\left[\max _{(s, l, c) \in \alpha, \alpha \in \mathcal{U}(h)} M(p, s, L, c) \leq M^{*}\right]\right\} .
$$

If, in addition to $M(\cdot) \leq M^{*}$, a limit on the variance of loss plus inspection costs is desired, then

$$
\begin{array}{r}
\hat{\alpha}(p)=\max \left\{h:\left[\max _{(s, l, c) \in \alpha, \alpha \in \mathcal{U}(h)} M(p, s, L, c) \leq M^{*}\right],\right. \\
\left.\left[\max _{(s, l, c) \in \alpha, \alpha \in \mathcal{U}(h)} V(p, s, L, c) \leq V^{*}\right]\right\} .
\end{array}
$$


TABle 1: Frequency distribution of boxes per shipment subject to AQI inspections, United States maritime ports, 2004-2006. Source: United States Department of Agriculture [38].

\begin{tabular}{lcc}
\hline Boxes per shipment & Shipments & Percent \\
\hline $0-500$ & 23 & $2.6 \%$ \\
$500-1000$ & 328 & $36.6 \%$ \\
$1000-1500$ & 385 & $42.9 \%$ \\
$1500-2000$ & 99 & $11.0 \%$ \\
$2000-2500$ & 34 & $3.8 \%$ \\
$2500-3000$ & 28 & $3.1 \%$ \\
\hline Total sample shipments & 897 & $100.0 \%$ \\
\hline
\end{tabular}

In either case, the optimal robust inspection strategy is the constant percentage of inspected containers $p^{*}=$ $\arg \max \hat{\alpha}(p)$.

\section{Agricultural Quarantine Inspections, Robustness, and Performance}

In this section we demonstrate the utility of the model by determining robust inspection protocols with data on various aspects of U.S. port inspections for invasives in fruit and vegetable shipments. We determine robust constant percentage inspection rules for shipping containers to compare to the current AQI protocol (the $2 \%$ inspection rule).

3.1. Approach and Data. For our simulations, we modify the problem so that our results will be in terms of the proportion of infested boxes instead of the number of infested boxes. This modification makes the interpretation of our results somewhat easier. Let $w$ be the proportion of infested boxes in a shipping container, and note that the corresponding number of infested items is $s=r(w N)$, where recall that $r$ indicates rounding to the nearest integer. Define the set $\boldsymbol{W}=$ $\{0, .01, .02, \ldots, 1\}$, that is, the set of discrete proportions in 0.01 unit increments from zero to 1 . Our simulation replaces the set of potential infested items $\&$ in the model development with the set $\boldsymbol{W}$.

Our first task is to construct an estimate of the mean and variance of expected loss plus inspection costs $((7)$ and (8)), modified so that potential infestations are in percentage terms. We take $f(N)$ from the relative frequency of boxes per container in a sample of 893 shipments subject to AQI inspection at maritime ports of entry in the United States from 2004-2006 (the most recent years for which data are available). This frequency distribution is shown in Table 1. Average total shipments subject to physical inspection under AQI during this period provides an estimate for $T$ of 496,265 shipments. See United States Department of Agriculture [38] for these data.

Although the model allows unit inspection costs to be uncertain, we developed a reasonable point estimate for this value. Regardless of port and/or commodity, a typical inspection costs approximately $\$ 1.70$ per box. This cost estimate is based on the typical time required to inspect fruit and vegetable commodities packaged in boxes and
TABle 2: Total USDA/APHIS emergency program funds, plant pests, 1987-2007*.

\begin{tabular}{cccc}
\hline Year & $\begin{array}{c}\text { Costs (millions of } \\
\text { 2007 dollars) }\end{array}$ & Year & $\begin{array}{c}\text { Costs (millions of } \\
\text { 2007 dollars) }\end{array}$ \\
\hline 1987 & 9.647 & 1998 & 36.343 \\
1988 & 6.940 & 1999 & 52.089 \\
1989 & 13.730 & 2000 & 176.562 \\
1990 & 44.096 & 2001 & 250.455 \\
1991 & 9.802 & 2002 & 93.523 \\
1992 & 26.914 & 2003 & 97.414 \\
1993 & 24.872 & 2004 & 117.779 \\
1994 & 27.497 & 2005 & 232.169 \\
1995 & 26.753 & 2006 & 470.883 \\
1996 & 48.249 & 2007 & 159.031 \\
1997 & 45.325 & & \\
\hline
\end{tabular}

* Important pests (in order of allocated funds): citrus canker, Mediterannean fruit fly, emeral ash borer, asian longhorned beetle, karnal bunt, Pierce's disease/GWSS, sudden oak death, asian gypsy moth, grasshopper and morman cricket, potato cyst nematode, Mexican/oriental/olive/west Indian fruit flies, plum pox virus, and others.

Source: United States Department of Agriculture [39].

the government service pay scale in effect for inspectors during 2009. (Personal communication, Rojelio Lozano, U.S. Department of Homeland Security). For our simulation exercise we take this inspection cost value as certain and specify the set $C$ as simply $C=\{1.70\}$. In contrast, the set of potential losses is highly uncertain. We specify the set of potential losses as $\mathcal{L}=\left\{0,1 \times 10^{6}, 2 \times 10^{6}, \ldots, 500 \times 10^{6}\right\}$.

We now turn to specifying the performance criteria, $M^{*}$ and $V^{*}$, in (10). Recall that the specifications of $M^{*}$ and $V^{*}$ are choice variables for the decision maker; they are not determined from the model, so they rely on the judgments of decision makers as to what constitutes acceptable performance. We use real USDA/APHIS annual emergency program expenditures, shown in Table 2 for 1987-2008, as a proxy for pest losses in the specification of possible performance criteria [39].(APHIS is the acronym for the USDA's Animal and Plant Health Inspection Service. We use the Producer Price Index averaged over fiscal years to convert nominal emergency funds to real values. The baseline year is 2007.) The amounts in the table are transfers from contingency funds to cover expenditures for unforeseen pest introductions or outbreaks that threaten agricultural production. These funds are not necessarily all due to inspection failures, nor do they include all pest losses to agricultural producers, landowners, and others. Thus, there is not a one-to-one correspondence between these values and potential losses from inspection failures. However, we think they are useful because the variation in annual expenditures allow us to specify a wide range of performance criteria. Moreover, these emergency fund expenditures give us a basis for casting our results in terms of the tradeoff between allocating resources to inspections to prevent introductions and allocating resources to deal with the consequences of inspection failures. 
We specify $M^{*}$ as an estimate of variable inspection costs under AQI plus alternative levels of emergency program expenditures from the distribution given in Table 2. For variable inspection costs, the density function $f(N)$ from the data in Table 1 combined with $T=496,265$ shipments gives us a mean for the annual number of boxes subject to AQI inspections of $586.306 \times 10^{6}$ with variance $846.593 \times 10^{26}$. These values, our estimate of variable per-box inspection costs of $\$ 1.70$ and the AQI rule of inspecting $2 \%$ of boxes in a container produces an estimated annual variable inspection cost of $\$(1.70) r\left(0.02 \times 586.306 \times 10^{6}\right) \approx 19.934 \times 10^{6}$, with variance $\$(1.70)^{2} r\left(0.04 \times 846.593 \times 10^{26}\right)$.

Our analysis is conducted with six levels of $M^{*}$. The variable inspection cost estimate is the same for each level, but we use six levels of damages based on real USDA/APHIS emergency expenditures. We use the mean of the distribution in Table 2 of $\$ 93.813 \times 10^{6}$, as well as the upper bounds of the quintiles of the distribution; $\$ 24.872 \times 10^{6}, \$ 36.343 \times 10^{6}$, $\$ 52.089 \times 10^{6}, \$ 159.031 \times 10^{6}$, and $\$ 470.883 \times 10^{6}$. In addition, we also specify $V^{*}$ as an additional performance criterion as in [10]. This value is set at the variance of annual inspection costs, specified above, plus the variance of the real USDA/APHIS emergency expenditures which is $\$ 12.786 \times$ $10^{9}$. The variance performance criterion is held constant as $M^{*}$ varies. For all of the results reported below, the variance performance criterion does not bind.

3.2. Results. Table 3 shows the optimal robust inspection rules and levels of robustness for alternative levels of performance in terms of $M^{*}$ as well as the robustness levels of the AQI $2 \%$ rule. In the table $M_{i}^{*}, i=1, \ldots, 5$, are variable inspections costs plus the top boundary of the $i$ th quintile of the distribution of real USDA/APHIS emergency funds. $M_{\text {mean }}^{*}$ is variable inspection costs plus the mean of the distribution of real USDA/APHIS emergency funds.

The last two columns of Table 3 give the robustness measures of the optimal inspection rules and the AQI rule for the various performance criteria. Recall that robustness is the cardinality of the largest element of the power set of the cross product of $\mathcal{W}$ and $\mathcal{L}$ under which a performance criterion is met. Thus, robustness is the number of potential outcomes in terms of discrete losses from undetected infestations and infestation percentages for which the performance criterion is met. For all performance criteria analyzed (except for $M_{\mathrm{AQI}}^{*}$ which will be explained shortly), robustness under the optimal inspection rule is greater than under the AQI rule. This, of course, is by design because the optimal inspection rule maximizes robustness. It is more interesting that the optimal inspection rules are much more robust than the AQI rule; robustness under the optimal inspection rule ranges from more than 2.36 times greater than under the AQI rule for $M_{1}^{*}$, to nearly 4.5 times greater for $M_{4}^{*}$. At $M_{\text {mean }}^{*}$ the optimal inspection strategy is about 4.12 times as robust as the AQI 2\% inspection rule. The AQI rule, which recall is devoid of the economic and uncertainty characteristics of the problem of detecting invasive species, is simply not very robust over a wide range of reasonable levels of economic performance.

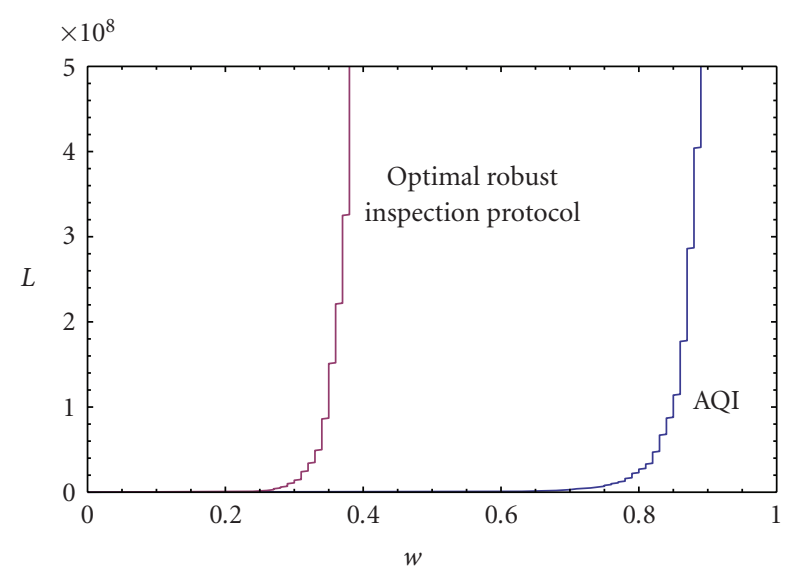

Figure 1: "Safety Zones" of optimal robust inspections and AQI Inspections.

A graphical depiction of the relative robustness of the optimal inspection protocol and the AQI inspection protocol is provided in Figure 1. The robustness "curves" in the figure are derived under the assumption that the performance criterion is $M_{\text {mean }}^{*}$. The left curve collects pairs of infestation rates and potential losses $(w, L)$ for which $M_{\text {mean }}^{*}$ is met exactly under the robust optimal inspection rate of $11 \%$. Pairs of $(w, L)$ to the right of this curve also meet the performance requirement under this inspection rule. We can think of the curve and the area to its right as a sort of "safety zone" in the sense that the decision maker is certain that the performance requirement is satisfied for all $(w, L)$ outcomes in this zone. The right curve in Figure 1 is the boundary of the safety zone for the $M_{\text {mean }}^{*}$ performance requirement, but under the AQI inspection rule of $2 \%$. Given our limit on $L$ (\$500 million), it is clear that the safety zone under the optimal robust inspection rule is much larger than under the AQI rule.

In addition, the safety zone under the AQI inspection rule is a proper subset of the safety zone under the robust optimal inspection rule. This is a desirable attribute because it implies that every outcome that meets the performance criterion under the AQI inspection strategy also meets the performance criterion under the robust optimal inspection strategy. We caution the reader that this is not a general result, that is, it is possible that the robustness curves could cross for some parameters of the problem or for some other application.

Let us now return to the results in Table 3. The change in maximum robustness levels for $M_{1}^{*}, \ldots, M_{5}^{*}$ reveals a fundamental tradeoff between robustness and performance: robustness decreases with a more stringent performance requirement (i.e., lower $M^{*}$ in this application). This reflects the fact that the circumstances under which a performance criterion is met are fewer if we insist on better performance. Although not seen in Table 3 it is easy to demonstrate that robustness is zero for $M^{*}=0$ indicating that a decision maker has no confidence in limiting expected costs to zero. Greater robustness only comes from tolerating higher expected costs. 
TABle 3: AQI and Optimal Robust Inspection Rules.

\begin{tabular}{ccccc}
\hline $\begin{array}{c}\text { Performance criterion } \\
\text { (Millions\$) }\end{array}$ & Optimum & AQI & Optimum & Robustness \\
\hline$M_{\text {mean }}^{*}=113.747$ & $11 \%$ & $2 \%$ & 33027 & 7961 \\
$M_{1}^{*}=44.806$ & $4 \%$ & $2 \%$ & 15106 & 6394 \\
$M_{2}^{*}=56.277$ & $5 \%$ & $2 \%$ & 18826 & 6766 \\
$M_{3}^{*}=72.023$ & $7 \%$ & $2 \%$ & 24694 & 7173 \\
$M_{4}^{*}=178.965$ & $16 \%$ & $2 \%$ & 39195 & 8734 \\
$M_{5}^{*}=490.817$ & $47 \%$ & $2 \%$ & 47453 & 10484 \\
$M_{\mathrm{AQI}}^{*} \approx 22.286$ & $2 \%$ & $2 \%$ & 5063 & 5063 \\
\hline
\end{tabular}

The second and third columns of Table 3 reveal that the optimal inspection strategies for $M_{1}^{*}, \ldots, M_{5}^{*}$, and $M_{\text {mean }}^{*}$ involve more inspections than the AQI $2 \%$ rule. Thus, for this range of performance criteria, greater robustness is achieved by more intense inspections than under AQI. Noting that the optimal inspection rule is monotonic in the performance criteria for this application, we asked whether there is a performance criterion at which the $2 \%$ rule maximizes robustness. This performance criterion does exist and it is approximately $M_{\mathrm{AQI}}^{*} \approx \$ 22.286 \times 10^{6}$. Given variable inspection costs of about $\$ 19.934 \times 10^{6}, M_{\mathrm{AQI}}^{*}$ allows for emergency funds of only $\$ 2.352 \times 10^{6}$. Note that this is lower than all of the emergency fund allocations in Table 2. This suggests that the AQI inspection rule is optimally robust only for unrealistically low performance criteria.

The data in Table 3 also reveal important information about how robustness is achieved with inspections. For now, focus on one performance criterion, say $M_{\text {mean }}^{*}$. That optimal inspections are substantially higher than AQI inspections under this performance criterion indicates that expected losses from introductions must be significantly less to hold variable inspection costs plus expected introduction losses to $M_{\text {mean }}^{*}$. The higher inspection percentage associated with the optimal robust protocol enables detections of much lower infestations, which reduces the chance of inspection failure and the expected losses due to inspection failure. The cost saving due to preventing inspection failure more than compensates for the added inspection cost. The tradeoff between lower expected introduction losses and higher inspections costs can be dramatic. At the performance criterion $M_{\text {mean }}^{*}$, the variable costs of optimal inspections $(11 \%)$ total $\$ 109.639 \times 10^{6}$ and expected emergency fund expenditures are only $\$ 4.108 \times 10^{6}$, or about $3.7 \%$ of the performance criterion. Under the $2 \%$ inspection rule with variable inspection costs of $\$ 19.934 \times 10^{6}$, expected emergency fund allocations are limited to $\$ 93.813 \times 10^{6}$ under $M_{\text {mean }}^{*}$, or about $82.5 \%$ of the performance criterion. As noted above, the former inspection strategy is much more robust than the AQI strategy. Thus, robust optimality appears to call for a shift in resources toward more inspections and away from emergency fund allocations to deal with invasive species introductions. That optimal inspection rates increase with the performance criterion in Table 3 is a further reflection of how maximizing robustness calls for shifting expenditures to more inspections. We should note, however, that the relative proportions of inspection costs and expected emergency expenditures do not change monotonically as the performance criterion is increased. In general, it is worth noting that the comparative static results in Table 3 are limited to our simulation exercise and should not be taken as general results.

\section{Conclusion}

We have proposed a protocol for determining inspection strategies for detecting invasive species in shipments of fruits and vegetables that considers the costs of inspections and potential losses from undetected introductions, but does not rely on probability distributions that real decision makers often lack. This protocol is to choose an inspection strategy, that is, robust in the sense that it maximizes the set of possible outcomes under which a performance criterion is met. We use this protocol to evaluate the robustness of the current practice of inspecting $2 \%$ of items in shipping containers of fruits and vegetables at U.S. ports. For a wide range of performance criteria the $2 \%$ rule is simply not very robust to the substantial uncertainty that characterizes the problem of preventing some invasive species introductions.

Moreover, our calculations of robust optimal inspection rules suggests that a shift of resources toward more inspections and away from allocating funds to deal with invasives that get past the inspection process may be justified. Currently, agricultural inspections are funded from AQI user fees collected when international passengers and conveyances (trucks, commercial vessels, rail cars, and aircraft) enter the United States. These fees are split between DHS and USDA's Animal and Plant Health Inspection Service (APHIS), with DHS receiving about 60 percent. The U.S. Government Accountability Office [40] estimated that the current cost of inspecting commercial vessels is considerably less than total fees collected. Increasing the agricultural inspection rate would require that a greater proportion of AQI user fees be allocated to inspections or an increase in funding to cover the costs of the greater workload. An increase in funding could require congressional action to increase budget appropriations or identify alternative funding sources.

Our protocol for determining robust optimal inspection rules can be used to address important issues about agricultural inspections that the U.S. Government Accountability Office has raised over the years. Since the protocol can be 
applied to poorly understood or surreptitiously introduced organisms for which probabilities are not easily available, it can address concerns about detecting new pests and agro-terrorism threats during inspection (United States Government Accountability Office [41] and United States Government Accountability Office [42]). By suggesting higher sampling rates than currently used under a wide range of performance criteria, the protocol can address concerns about inspection reliability raised by declining interceptions as import shipments increased [41-43].

Although our work is motivated by detecting invasive species in fruit and vegetable shipments, this same approach is applicable to an increasingly wide range of detection problems where uncertainty is severe and effective use of scarce inspection resources is required. Useful applications of this approach likely include border inspections for smuggled contraband, general law enforcement problems, and the early detection and control of infectious diseases.

\section{Acknowledgment}

Funding for this research was provided by the U. S. Department of Agriculture under USDA/ERS/PREISM Cooperative Agreement no. 43-3AEM-4-80115. Additional support was provided by the Cooperative State Research Extension, Education Service, U. S. Department of Agriculture, Massachusetts Agricultural Experiment Station under Project no. MAS00861. The views expressed in the paper are the authors' and do not necessarily represent those of the sponsoring agencies. The authors are grateful to the United States Department of Homeland Security, particularly National Data Quality Manager, Rojelio Lozano, for providing unpublished information on the Agricultural Quarantine Inspection program used in this research. Without implicating them, this article has benefited from the helpful input of Yakov Ben-Haim, Barry C. Field, and Peyton M. Ferrier.

\section{References}

[1] United States Department of Agriculture, Animal and Plant Health Inspection Service, "Regulating the Importation of Fresh Fruits and Vegetables," 2008, http://www.aphis.usda .gov/import_export/plants/manuals/ports/downloads/fv.pdf.

[2] L. J. Moffitt and C. D. Osteen, "Prioritizing invasive species threats under uncertainty," Agricultural and Resource Economics Review, vol. 35, no. 1, pp. 41-51, 2006.

[3] J. K. Stranlund and Y. Ben-Haim, "Price-based vs. quantitybased environmental regulation under Knightian uncertainty: an info-gap robust satisficing perspective," Journal of Environmental Management, vol. 87, no. 3, pp. 443-449, 2008.

[4] B. Render, R. M. Stair Jr., and M. E. Hanna, Quantitative Analysis for Management, Pearson Education, Upper Saddle River, NJ, USA, 10th edition, 2009.

[5] D. W. Katzner, Time, Ignorance, and Uncertainty in Economic Models, The University of Michigan Press, Ann Arbor, Mich, USA, 1998.

[6] R. D. Horan, C. Perrings, F. Lupi, and E. H. Bulte, "Biological pollution prevention strategies under ignorance: the case of invasive species," American Journal of Agricultural Economics, vol. 84, no. 5, pp. 1303-1310, 2002.
[7] I. Gilboa and D. Schmeidler, "Maxmin expected utility with non-unique prior," Journal of Mathematical Economics, vol. 18, no. 2, pp. 141-153, 1989.

[8] Y. Ben-Haim, Info-Gap Decision Theory: Decisions Under Severe Uncertainty, Academic Press, Amsterdam, The Netherlands, 2nd edition, 2006.

[9] Y. Ben-Haim, "Value at risk with info-gap uncertainty," Journal of Risk Finance, vol. 6, pp. 388-403, 2005.

[10] Y. Carmel and Y. Ben-Haim, "Info-gap robust-satisficing model of foraging behavior: do foragers optimize or satisfice?" American Naturalist, vol. 166, no. 5, pp. 633-641, 2005.

[11] B. S. Halpern, H. M. Regan, H. P. Possingham, and M. A. McCarthy, "Accounting for uncertainty in marine reserve design," Ecology Letters, vol. 9, no. 1, pp. 2-11, 2006.

[12] A. Moilanen and B. A. Wintle, "Uncertainty analysis favours selection of spatially aggregated reserve networks," Biological Conservation, vol. 129, no. 3, pp. 427-434, 2006.

[13] L. J. Moffitt, J. K. Stranlundt, and B. C. Field, "Inspections to avert terrorism: robustness under severe uncertainty," Journal of Homeland Security and Emergency Management, vol. 2, no. 3, article 3, 2005.

[14] L. J. Moffitt, J. K. Stranlund, B. C. Field, and C. D. Osteen, "Robust inspection for invasive species with a limited budget," in New Approaches in the Economics of Plant Health, A. O. Lansink, Ed., Springer, Amsterdam, The Netherlands, 2007.

[15] L. J. Moffitt, J. K. Stranlund, and C. D. Osteen, "Robust detection protocols for uncertain introductions of invasive species," Journal of Environmental Management, vol. 89, no. 4, pp. 293-299, 2008.

[16] L. Davidovitch, R. Stoklosa, J. Majer et al., "Info-gap theory and robust design of surveillance for invasive species: the case study of Barrow Island," Journal of Environmental Management, vol. 90, no. 8, pp. 2785-2793, 2009.

[17] T. M. Rout, C. J. Thompson, and M. A. McCarthy, "Robust decisions for declaring eradication of invasive species," Journal of Applied Ecology, vol. 46, no. 4, pp. 782-786, 2009.

[18] M. A. Burgman, B. A. Wintle, C. A. Thompson, A. Moilanen, M. C. Runge, and Y. Ben-Haim, "Reconciling uncertain costs and benefits in bayes nets for invasive species management," Risk Analysis, vol. 30, no. 2, pp. 277-284, 2010.

[19] L. R. Carrasco, R. Baker, A. MacLeod, J. D. Knight, and J. D. Mumford, "Optimal and robust control of invasive alien species spreading in homogeneous landscapes," Journal of the Royal Society Interface, vol. 7, no. 44, pp. 529-540, 2010.

[20] D. Yemshanov, F. H. Koch, Y. Ben-Haim, and W. D. Smith, "Robustness of risk maps and survey networks to knowledge gaps about a new invasive pest," Risk Analysis, vol. 30, no. 2, pp. 261-276, 2010.

[21] P. Vinot, S. Cogan, and V. Cipolla, "A robust model-based test planning procedure," Journal of Sound and Vibration, vol. 288, no. 3, pp. 571-585, 2005.

[22] C. D. Osteen, E. B. Bradley, and L. J. Moffitt, The Economics of Agricultural Pest Control: An Annotated Bibliography, 19601980, United States Department of Agriculture, Economic Research Service, Bibliographies and Literature of Agriculture Series no. 14, 1981.

[23] J. M. White, P. G. Allen, L. J. Moffitt, and P. P. Kingsley, "Economic analysis of an areawide program for biological control of the alfalfa weevil," American Journal of Alternative Agriculture, vol. 10, no. 4, pp. 173-179, 1995.

[24] D. Pimentel, L. Lach, R. Zuniga, and D. Morrison, "Environmental and economic costs of nonindigenous species in the United States," BioScience, vol. 50, no. 1, pp. 53-65, 2000. 
[25] C. Perrings, M. Williamson, and S. Dalmazzone, Eds., The Economics of Biological Invasions, Edward Elgar, Cheltenham, UK, 2000.

[26] J. F. Shogren, "Risk reduction strategies against the 'explosive invader", in The Economics of Biological Invasions, M. Perrings, M. Williamson, and S. Dalmazzone, Eds., pp. 56-69, Edward Elgar, Cheltenham, UK, 2000.

[27] C. Brown, L. Lynch, and D. Zilberman, "The economics of controlling insect-transmitted plant diseases," American Journal of Agricultural Economics, vol. 84, no. 2, pp. 279-291, 2002.

[28] E. B. Barbier and J. F. Shogren, "Growth with endogenous risk of biological invasion," in Proceedings of the 77th Annual Western Agricultural Economic Association International Conference, Seattle, Wash, USA, 2002.

[29] M. E. Eiswerth and G. C. Van Kooten, "Uncertainty, economics, and the spread of an invasive plant species," American Journal of Agricultural Economics, vol. 84, no. 5, pp. 13171322, 2002.

[30] L. H. Endress, "Terrorism and the economics of biological invasions," in Proceedings of the 77th Annual Western Agricultural Economic Association International Conference, Seattle, Wash, USA, 2002.

[31] B. Kaiser and J. Roumasset, "Optimal public control of exotic species: preventing the brown tree snake from invading Hawaii," in Proceedings of the 77th Annual Western Agricultural Economic Association International Conference, Seattle, Wash, USA, 2002.

[32] L. J. Olson and S. Roy, "The economics of controlling a stochastic biological invasion," American Journal of Agricultural Economics, vol. 84, no. 5, pp. 1311-1316, 2002.

[33] C. Perrings, M. Williamson, E. B. Barbier et al., "Biological invasion risks and the public good: an economic perspective," Conservation Ecology, vol. 6, no. 1, 2002.

[34] C. Settle and J. F. Shogren, "Modeling native-exotic species within Yellowstone Lake," American Journal of Agricultural Economics, vol. 84, no. 5, pp. 1323-1328, 2002.

[35] C. McAusland and C. Costello, "Avoiding invasives: traderelated policies for controlling unintentional exotic species introductions," Journal of Environmental Economics and Management, vol. 48, no. 2, pp. 954-977, 2004.

[36] I. V. Surkov, A. G. J. M. Oude Lansink, O. van Kooten, and W. van der Werf, "A model of optimal import phytosanitary inspection under capacity constraint," Agricultural Economics, vol. 38, no. 3, pp. 363-373, 2008.

[37] Y. Ben-Haim and K. W. Hipel, "The graph model for conflict resolution with information-gap uncertainty in preferences," Applied Mathematics and Computation, vol. 126, no. 2-3, pp. 319-340, 2002.

[38] United States Department of Agriculture, Animal and Plant Health Inspection Service, Unpublished Plant Protection and Quarantine-280 data, 2008.

[39] United States Department of Agriculture, Animal and Plant Health Inspection Service, Unpublished Agricultural Quarantine Inspection Monitoring data, 2008.

[40] United States Government Accountability Office, "Federal User Fees: Substantive Reviews Needed to Align Port-Related Fees with the Programs They Support," GAO-08-321, February 2008.

[41] United States Government Accountability Office, "Agricultural Quarantine Inspection: Management Problems May Increase Vulnerability of U.S. Agriculture to Foreign Pests and Diseases," GAO-08-96T, October 2007.
[42] United States Government Accountability Office, "Homeland Security: Much Is Being Done to Protect Agriculture from a Terrorist Attack, but Important Challenges Remain," GAO-05214, March 2005.

[43] United States General Accounting Office, "Agricultural Inspection: Improvements Needed to Minimize Threat of Foreign Pests and Diseases," GAO/RCED-97-102, May 1997. 


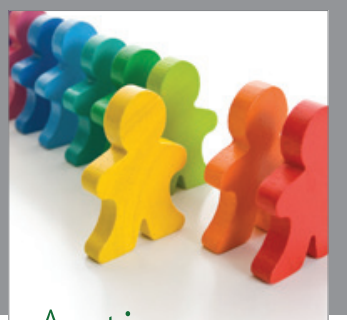

Autism

Research and Treatment
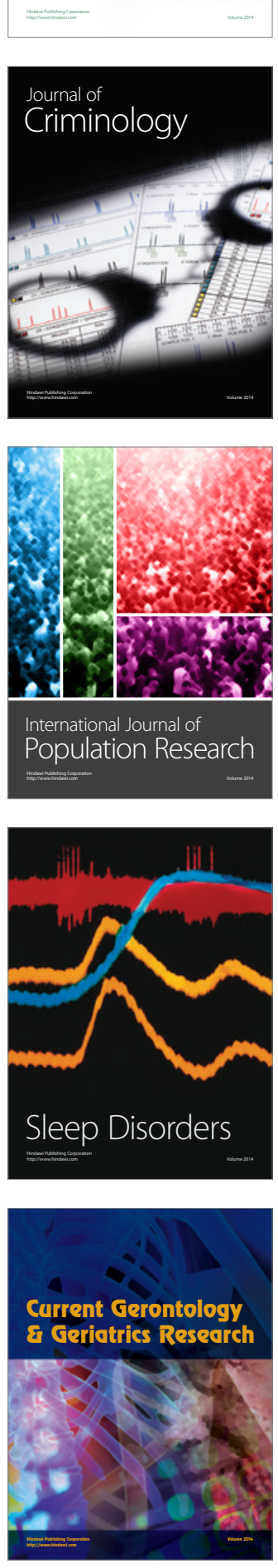
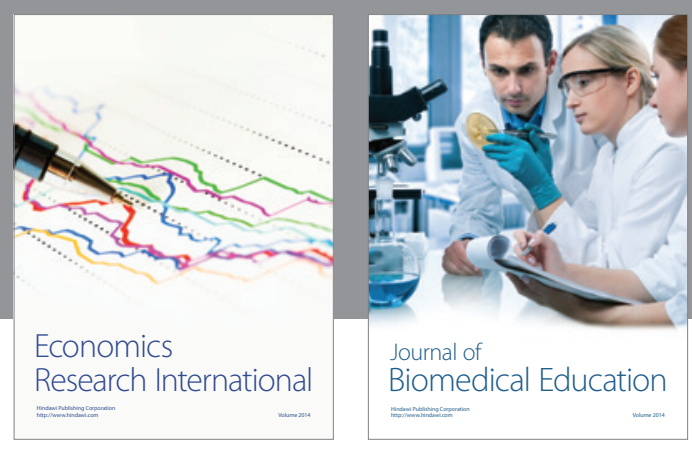

Journal of

Biomedical Education

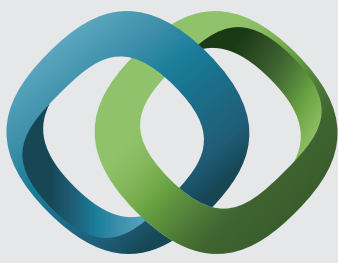

\section{Hindawi}

Submit your manuscripts at

http://www.hindawi.com
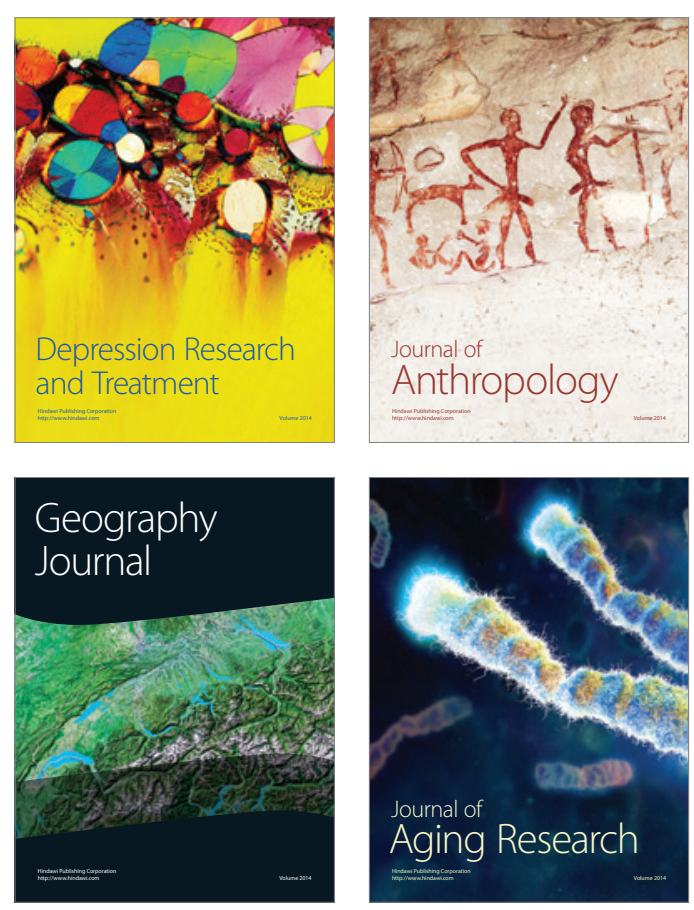

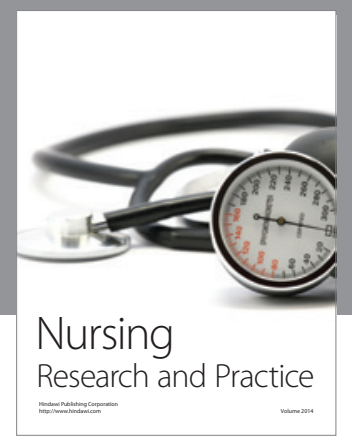

Nursing

Research and Practice

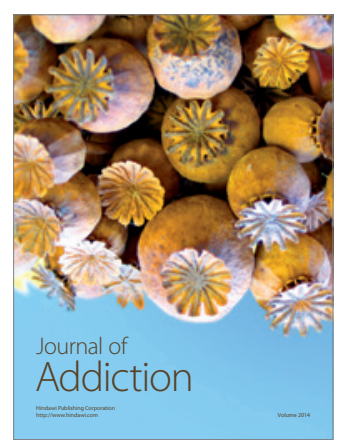

Child Development

Research

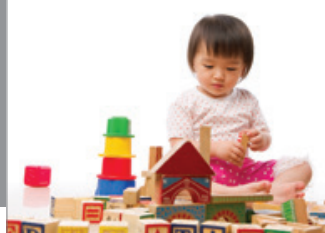

迥
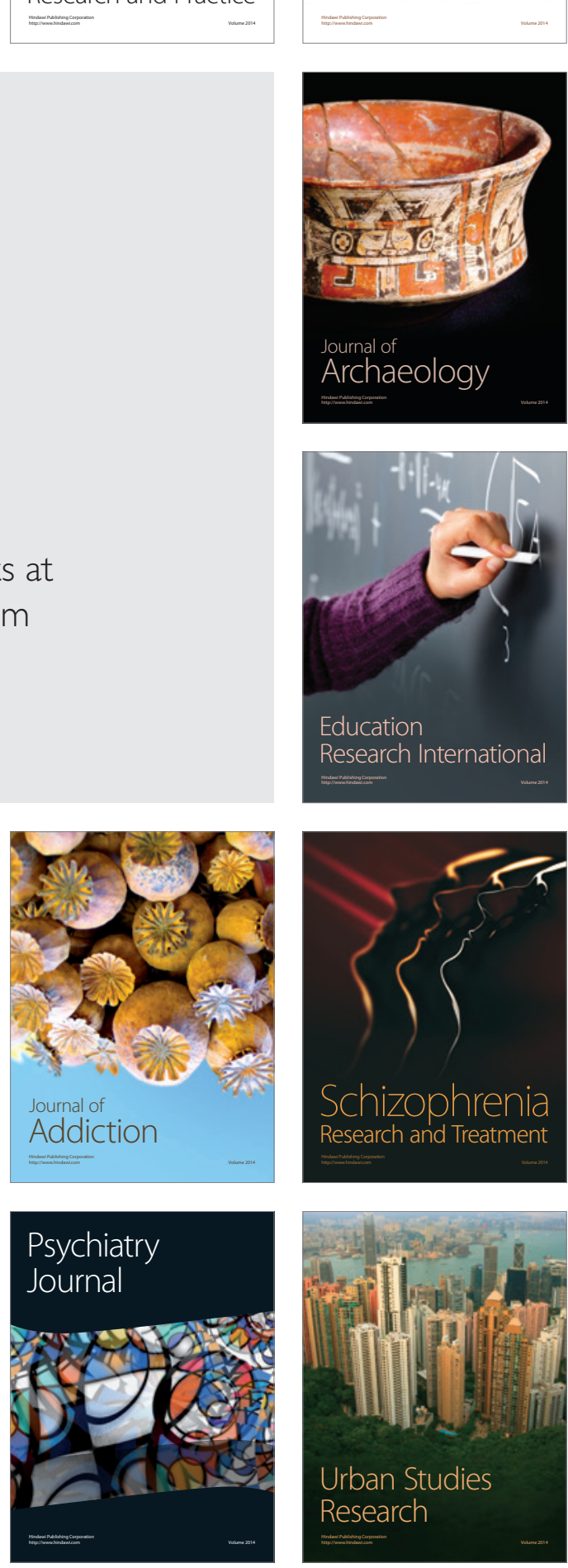